\title{
Study of soccer ball flight trajectory
}

\author{
Juliana Javorova ${ }^{1, *}$, Anastas Ivanov $^{2}$ \\ ${ }^{1}$ University of Chemical Technology and Metallurgy, Sofia, Bulgaria \\ ${ }^{2}$ Todor Kableshkov University of Transport, Sofia, Bulgaria
}

\begin{abstract}
In this paper the trajectories of a soccer ball for the most important kicks in the football game - a corner kick and a direct free kick are studied. The soccer ball is modelled as an ideal rigid hollow spherical body with six degrees of freedom, which performs a general motion in an immovable air environment with constant parameters. The ball 3D orientation is determined by the three Cardan angles. The aerodynamic forces and moments with which the air environment acts to the ball are taken into account. Two of the most dangerous areas of the football goal are defined. Differential equations which describe the motion of the soccer ball are solved numerically by MatLab-Simulink.
\end{abstract}

\section{Introduction}

Football is one of the most popular ball games. The footballer skills to give the soccer ball the necessary direction, speed and rotation is an extremely important quality that can be improved and perfected if the physical nature of the soccer ball motion in the air is known, as well as the factors that affect this flight.

In the last twenty years, there has been a strong rise in the researches and publications of various types of sports balls, in particular to balls for tennis, golf, baseball, football and volleyball [1-6, etc.]. And although there are many common laws among them, each ball has specific features.

The soccer ball, compared to the ones listed above, has got the largest dimensions and weight. It also differs from them in that its free motion is achieved by the players' feet and sport shoes. In the papers $[7,8$, etc.] the footstep of the footballer together with his shoe and the soccer ball are modelled by Finite Element Method.

Most of the studies can be grouped mainly in the following directions.

For one big part of papers it is assumed that the fluid environment is under steady state conditions and the soccer ball moves with a certain speed in it. Here the theoretical knowledge of aerodynamics is complemented and refined by modern experiments [9-14].

In the second group of researches [15-18, etc.] the ball is considered as an immovable and it is flowed by the moving fluid environment. These studies are classical and are related to the presence of a fluid tube (tunnel). Different aerodynamic coefficients are determined, in particular the drag coefficient and the lift force coefficient in presence and absence of ball rotation.

\footnotetext{
*Corresponding author: july@uctm.edu
} 
A mathematical study of the soccer ball aerodynamic interaction with the fluid environment based on the boundary layer theory is treated in details in $[19,20]$.

One important clarification of the effect of Magnus is done in the paper [21, 22].

In the researches [23-26] the most important kicks in the football game/direct free kick and corner kick/ are studied.

There are also many publications, sporting and methodical developments, in which the soccer ball strokes are studied from biomechanical point of view [27, 28].

The present study is directed namely to the above mentioned two soccer kicks. The main objectives of this paper can be formulated, as follows:

- To define the most dangerous zone in the upper left or right corner of the goal, which is farthest from the goalkeeper and therefore the ability to score is greatest;

- To find the initial linear and angular velocities also initial angles which are enough for soccer ball to go in the above-mentioned dangerous zone using two kind of soccer strokes;

- To illustrate the trajectories of potential successful kicks graphically.

\section{Characteristics of soccer balls and football fields}

The soccer balls used in professional leagues and in the FIFA and World Cup have five different sizes. Dimensions 1 and 2 are for promotional soccer balls, size 3 is used for children less than 8 years of age, while size 4 is used for young men and young women aged between 8 and 12 years. Size 5 is used for official football matches for men and women over 12 years of age.

Soccer ball size 5 is described by the following technical characteristics: circumference from 27 to $28 \mathrm{in}$ (from 69 to $71 \mathrm{~cm}$ ); diameter from 8.6 to 9 in (from 22 to $23 \mathrm{~cm}$ ); weight from 14 to $16 \mathrm{oz}$ (from 400 to $450 \mathrm{gr}$ ); internal pressure between 8.5 and 15.6 PSI (from $0.6 \times 10^{5}$ to $1.1 \times 10^{5} \mathrm{~Pa}$ ). The modern soccer balls have a different type of external relief and colours (Fig.1).

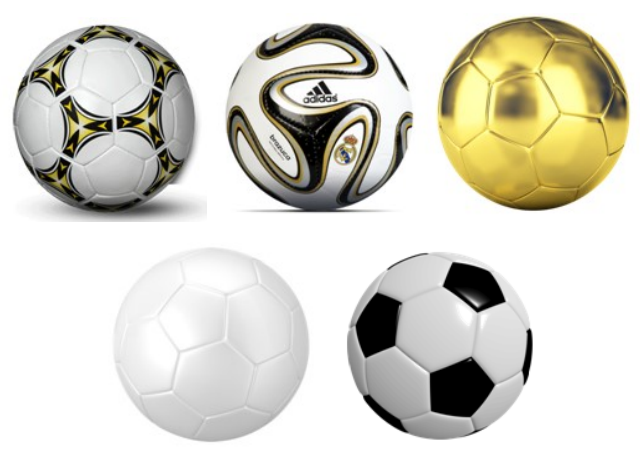

Fig. 1. Some professional soccer balls.

However, the most popular soccer ball consists of 12 pentagons and 20 hexagons from special artificial leather. They are joined together so that the soccer ball is an ideal sphere. The skin is extremely smooth and varnished. The connecting points of the individual leather pieces represent indentations which (analogical of the golf balls) further reduce the air environment resistance.

The football fields (called also football pitches or football grounds) are rectangular and usually vary in size. They have a length from 90 to $120 \mathrm{~m}$ and a width from 45 to $90 \mathrm{~m}$. 
For international matches after March 2008 the football pitches have official standard sizes. They are: $105 \mathrm{~m}$ long and $68 \mathrm{~m}$ wide. For example, the Allianz-Arena Stadium in Munich has just the same sizes. However, the grass covers a larger area. It is about $111 \mathrm{~m}$ long and $72 m$ wide (Fig. 2).

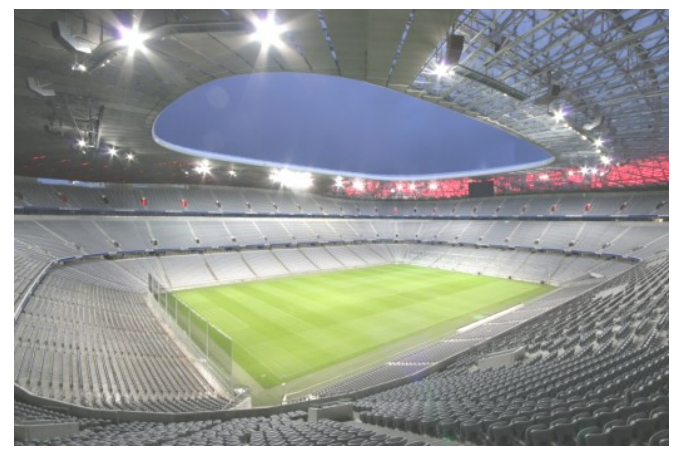

Fig. 2. Allianz-Arena Stadium in Munich ( http://sportslens.com/allianz-arena/92352/ ).

In the past football fields in South America was constructed with maximum sizes. Today, these playgrounds are rebuilt and now they have standard sizes. For example, these are the stadiums of Maracana (Rio de Janeiro), Antonio Vespuco Liberty and Jose Amalitani (Buenos Aires), Azteca (Mexico) and others.

\section{Mechanics of ball flight}

One of the basic assumptions related to the soccer ball mathematical model is that it is considered as a perfectly rigid sphere. Therefore in all of the time of football play, its dimensions remain constant.

\subsection{Kinematics}

The soccer ball has six degrees of freedom during the flight. It performs a general motion in the three-dimensional space. This motion can be counted with respect to a conditionally accepted motionless reference system $O x y z$.

Each general motion of a rigid body is complexly composed. It consists of a translation component that depends on the selected pole and a spherical component that does not depend on that choice.

The ball centre $C$ is selected for the pole. Therefore, the translation component of body motion is defined by the three functions of time (Fig.3):

$$
x=x(t), y=y(t), z=z(t) .
$$

In order to define the spherical component of the motion, two main reference systems are introduced. The first one $C X Y Z$ has the mass centre $C$ of the ball. It performs translational motion to the $O x y z$ system. The second one $C \xi \eta \zeta$ is invariably fixed to the ball and it is called movable reference system. Moreover, for describing of the spherical motion, two additional reference systems $C x_{1} y_{1} z_{1}$ and $C x_{2} y_{2} z_{2}$ are used. The orientation of the axes $C \xi, C \eta$ and $C \zeta$ in the space is defined by the three Cardan angles: rotation to an angle $\psi(t)$ around the axis $X \equiv x_{1}$, rotation to an angle $\theta(t)$ 
around the axis $y_{1} \equiv y_{2}$, and finally, rotation to an angle $\varphi(t)$ around the axis $z_{2} \equiv \zeta$. Therefore, the spherical component of the body motion is defined by the three functions:

$$
\psi=\psi(t), \theta=\theta(t), \varphi=\varphi(t) .
$$

By functions (1) and (2) the law of general motion of the soccer ball is defined.

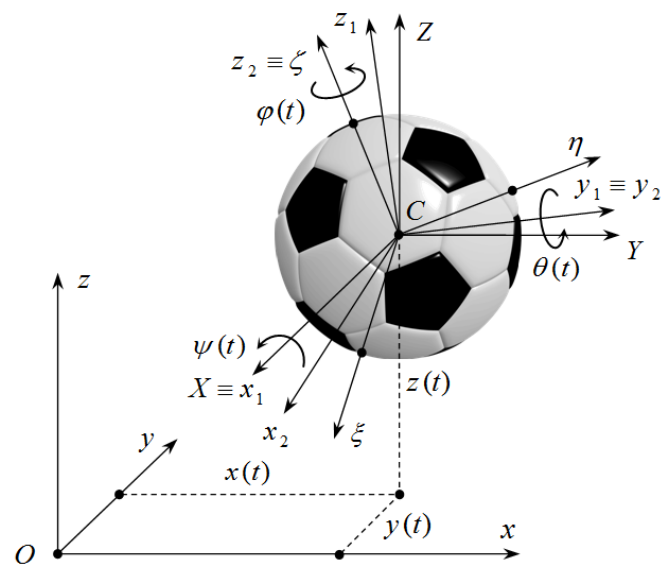

Fig. 3. Generalized coordinates of the soccer ball describing its position in the space.

The soccer ball angular velocity $\omega(t)$ can be determined by its algebraic projections with respect to the axes of $C X Y Z$ system. These formulas are the so-called kinematic Cardan equations and they have the following form:

$$
\begin{gathered}
\omega_{X}=\dot{\psi}+\dot{\varphi} \cdot \sin \theta, \\
\omega_{Y}=\dot{\theta} \cdot \cos \psi-\dot{\varphi} \cdot \cos \theta \cdot \sin \psi, \\
\omega_{Z}=\dot{\varphi} \cdot \cos \theta \cdot \cos \psi+\dot{\theta} \cdot \sin \psi .
\end{gathered}
$$

\subsection{Aerodynamics}

The following assumptions are introduced in advance. The air environment has steady-state parameters and the influence of the wind actions is not taken into account.

It is known that the complex aerodynamic interaction between the air and the flying sport ball can be analysed analytically by the boundary layer theory. However, in the practice only the main conclusions of this theory are used, as primarily all is based on the experimental researches. The latter are imposed because of very complicated mathematical description of this aerodynamic interaction as well as the great variety of the sports (in particular soccer) balls that does not allow unification of equations.

The main force acting on the ball is the force of gravity (Fig. 4):

$$
\mathbf{G}=m \cdot \mathbf{g},
$$

where $m$ is the soccer ball mass, $\mathbf{g}$ is a gravitational acceleration. 
The second force acting on the ball is the force of Archimedes. It is directly opposite to the force of gravity and is determined by the formula:

$$
\mathbf{W}_{A}=-(0.40) \cdot \pi \cdot R^{3} \cdot \rho_{a} \cdot \mathbf{g}
$$

where $R=0.11 \mathrm{~m}$ is the ball mean radius, $\rho_{a}=1.205 \mathrm{~kg} / \mathrm{m}^{3}$ is the air density at temperature about $t=20^{\circ} \mathrm{C}$.

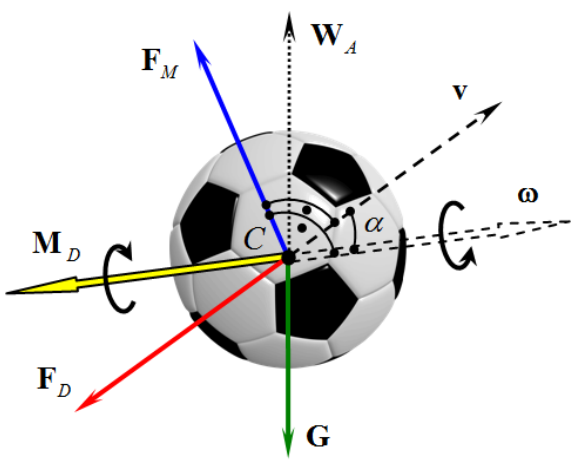

Fig. 4. Forces acting on the soccer ball during its flight in the air

The next force that acts on the soccer ball is a drag force. Its action is in the opposite direction of the velocity of the ball centre of mass (Fig. 4). This force depends on the square of that velocity and it is determined by the formula:

$$
\mathbf{F}_{D}=-\frac{1}{2} \cdot C_{D} \cdot \rho_{a} \cdot A \cdot v^{2} \cdot \frac{\mathbf{v}}{|\mathbf{v}|},
$$

where $A=\pi \cdot R^{2}$ is the middle cross-section area of the ball, $C_{D}$ is a drag coefficient.

The values of the non-dimensional drag coefficient depend on the kind of motion of the flying soccer ball. For the case of a translation without spin, for example straight central stroke during the football play, this coefficient $C_{D}$ depends mainly on the Reynolds number $\operatorname{Re}=v \cdot D / \vartheta$. The coefficient of kinematic viscosity is $\vartheta=1.50 \times 10^{-5} \mathrm{~m}^{2} / \mathrm{s}$ at environment temperature $t=20{ }^{\circ} \mathrm{C}$. Then, at the soccer ball velocity in the range $v=20 \div 30 \mathrm{~m} / \mathrm{s}$, the Reynolds number ranges from $R e=2.93 \times 10^{5}$ to $R e=4.40 \times 10^{5}$. The $R e$ values show that the motion performs under turbulent conditions, (Fig. 5).

From Fig. 5 it can be seen that for the above-mentioned values of the velocity $v$ and Reynolds number $R e$, the drag coefficient in the absence of rotation is approximately equal to $C_{D}=0.20$.

If the soccer ball motion has a rotational component also together with translational one, the drag coefficient depends both on the Reynolds number $R e$ and on the spin factor $S_{p}=\omega \cdot R / \vartheta$. In order to obtain the appropriate rotation of the ball, it is necessary the angular velocity to be within the following limits $\omega=60 \div 120 \mathrm{~s}^{-1}$. Therefore, the spin factor values must be ranged as $S_{p}=0.33 \div 0.44$. 
As it can be seen on Fig. 6, for the given above limits of values of the Reynolds number $R e$ and spin factor $S_{p}$ the drag coefficient must vary in the range $C_{D}=0.30 \div 0.35$.

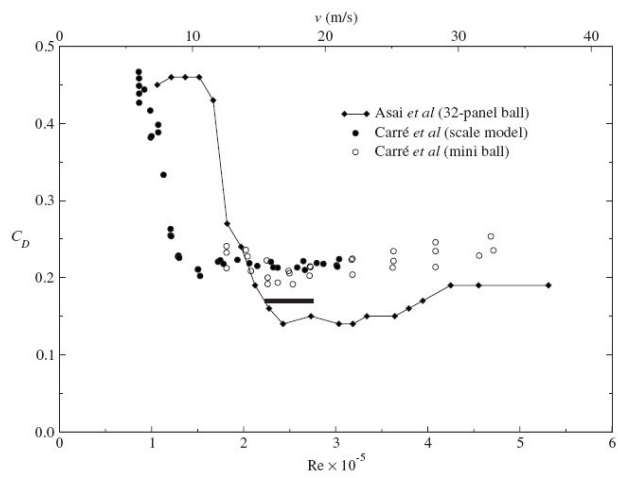

Fig. 5. Wind-tunnel data for $C_{D}$ as a function of $R e$ and $v$, according to [13].

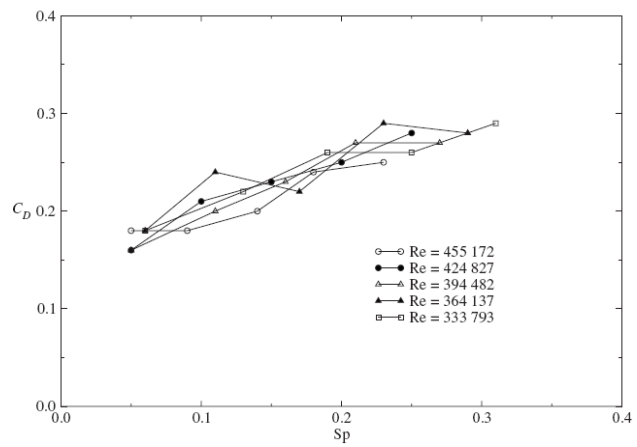

Fig. 6. Wind-tunnel data for $C_{D}$ as a function of $S_{p}$, according to [13].

The fourth force that acts on the soccer ball is the lift force which is very often called Magnus force (Fig.4). It also depends on the square of velocity but it acts in the direction of the vector cross-product $\omega \times \mathbf{v}$ :

$$
\mathbf{F}_{M}=\frac{1}{2} \cdot C_{L} \cdot \rho_{a} \cdot A \cdot v^{2} \cdot \frac{\omega \times \mathbf{v}}{|\omega \times \mathbf{v}|} .
$$

Here the dimensionless lift coefficient $C_{L}$ also depends on the Reynolds number $R e$ and on the spin factor $S_{p}$. From the results presented in [14] it can be concluded that for the above mentioned limits of $R e$ and $S_{p}$ the coefficient $C_{L}$ values must be ranged as follows $C_{L}=0.25 \div 0.35$.

Furthermore, in order to be the aerodynamic study more accurate, it is also necessary to introduce the so-called drag moment. It has to act in the opposite direction of the angular velocity $\omega$ at the presumption that the investigated soccer ball is an ideal sphere:

$$
\mathbf{M}_{D}=-\frac{1}{2} \cdot C_{D}^{(m)} \cdot \rho_{a} \cdot A \cdot v^{2} \cdot \frac{\omega}{|\omega|} .
$$

It was not found references with experimental data for determination of the coefficient $C_{D}^{(m)}$. By reason of this and because the soccer ball is quite smooth and polished (at least at the beginning of the game), it can be concluded that this coefficient has comparatively low values. Therefore, in the present study it was accepted for $C_{D}^{(m)}=0.05$.

\section{Differential equations}

The differential equations, which describes the three dimensional general motion of a soccer ball, have the following form: 


$$
\begin{gathered}
m . \ddot{x}=-k_{D} \cdot \dot{x} \cdot \sqrt{\dot{x}^{2}+\dot{y}^{2}+\dot{z}^{2}}+k_{L} \cdot\left(\omega_{Y} \cdot \dot{z}-\omega_{Z} \cdot \dot{y}\right), \\
m \cdot \ddot{y}=-k_{D} \cdot \dot{y} \cdot \sqrt{\dot{x}^{2}+\dot{y}^{2}+\dot{z}^{2}}+k_{L} \cdot\left(\omega_{Z} \cdot \dot{x}-\omega_{X} \cdot \dot{z}\right), \\
m . \ddot{z}=-k_{D} \cdot \dot{z} \cdot \sqrt{\dot{x}^{2}+\dot{y}^{2}+\dot{z}^{2}}+k_{L} \cdot\left(\omega_{X} \cdot \dot{y}-\omega_{Y} \cdot \dot{x}\right), \\
J \cdot \dot{\omega}_{X}=-k_{D}^{(m)} \cdot \omega_{X}, \\
J \cdot \dot{\omega}_{Y}=-k_{D}^{(m)} \cdot \omega_{Y}, \\
J \cdot \dot{\omega}_{Z}=-k_{D}^{(m)} \cdot \omega_{Z} .
\end{gathered}
$$

These equations must be solved together with the kinematic equations (3), (4), (5) at the respective initial conditions. Introduced above auxiliary multipliers can be written as:

$$
\begin{gathered}
k_{D}=\frac{1}{2} \cdot C_{D} \cdot \rho_{a} \cdot A \\
k_{L}=\frac{1}{2} \cdot C_{L} \cdot \rho_{a} \cdot A \cdot\left(\dot{x}^{2}+\dot{y}^{2}+\dot{z}^{2}\right) \cdot \frac{1}{|\boldsymbol{\omega} \times \mathbf{v}|}, \\
k_{D}^{(m)}=\frac{1}{2} \cdot C_{D}^{(m)} \cdot \rho_{a} \cdot A \cdot \frac{1}{|\boldsymbol{\omega}|} .
\end{gathered}
$$

\section{Goal target and soccer kicks modelling}

\subsection{Goal target}

To all footballers including the goal keepers are well known the two most dangerous zones of the goal. If the soccer ball goes to these areas, the probability of a score is extremely high. These are small areas around the upper two corners (left and right) of each goal, as in the current paper they are defined as a quarter of an ellipse whose centre is located in the inner corner of the goal (Fig. 7). The main dimensions of this ellipse are, as follows:

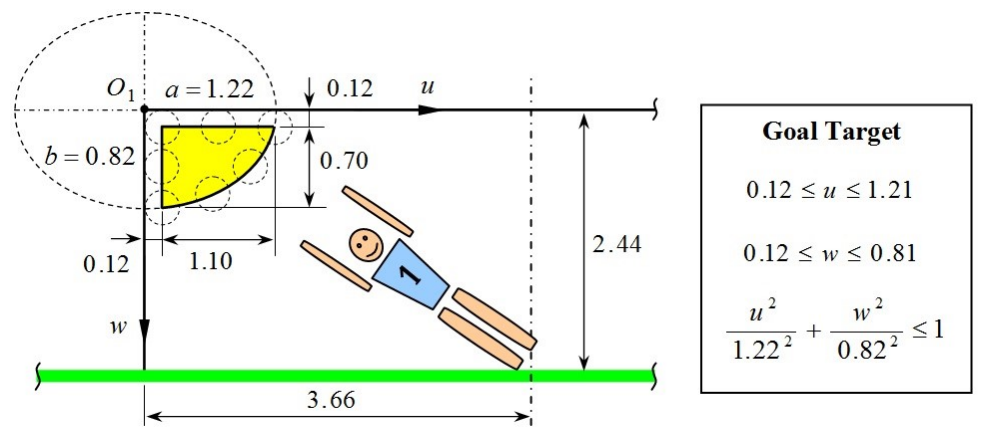

Fig. 7. Goal target - most dangerous zone for goal keeper.

long half-axis $a=1.22 \mathrm{~m}$, short half-axis $b=0.82 \mathrm{~m}$. Two strips (horizontal and vertical) of width $c=0,12 \mathrm{~m}$ are removed from the considered part of the ellipse as the size $c$ is 
chosen to be larger than the maximum radius $R$ of all models of soccer balls. In the paper namely above-mentioned parts of ellipse are regarded as a goal target (left or right).

\subsection{Corner kick}

Usually, when a corner kick is performed, the football striker kicks the ball strongly and high so that it reaches the penalty area where his teammates are located. There they attempt to control the falling ball and by head or by foot shot (volley, back scissors or a simple kick) try to direct the ball to the opponent's goal. So, the classic implementation of the corner kick is composed by two separated but connected each other elements.
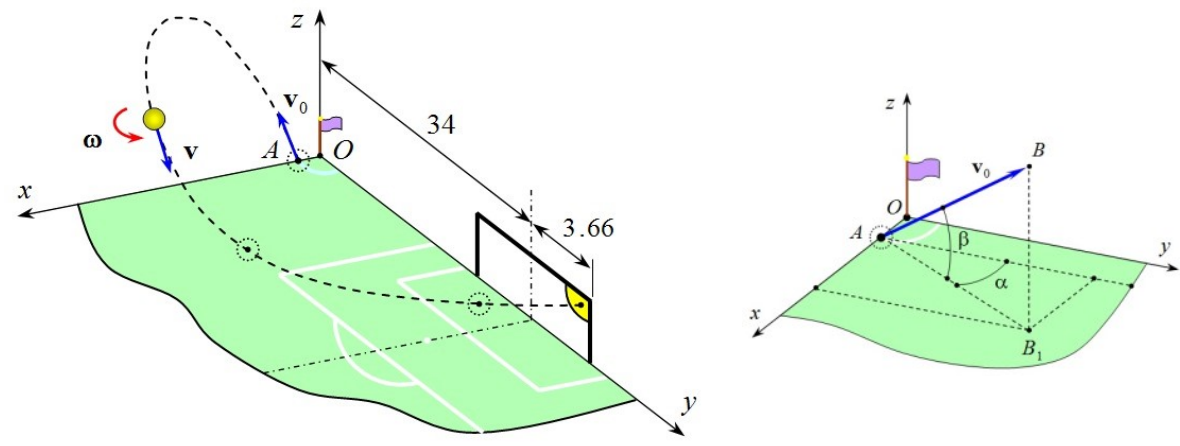

Fig. 8. Corner kick.

Current paper deals with another popular case about a corner kick. The corner striker gives the soccer ball such an initial linear and angular velocity that, thanks to the Magnus effect, the ball flies over half the width of the football field as deviating from the straight line trajectory and finally goes exactly to the remote upper corner of the opponent's goal (Fig. 8). In this way, it is achieved a direct score by the corner kick.

\subsection{Free kick}

In the paper is considered a direct free kick that aims to the score. The starting point from which the footballer performs the free kick (point $O$ on Fig. 9) is located at a distance $20 \mathrm{~m}$ from the goal line on the direction of the central longitudinal line of the football pitch. A wall of defenders is built at a distance $9.14 \mathrm{~m}$ on the same direction. It is assumed
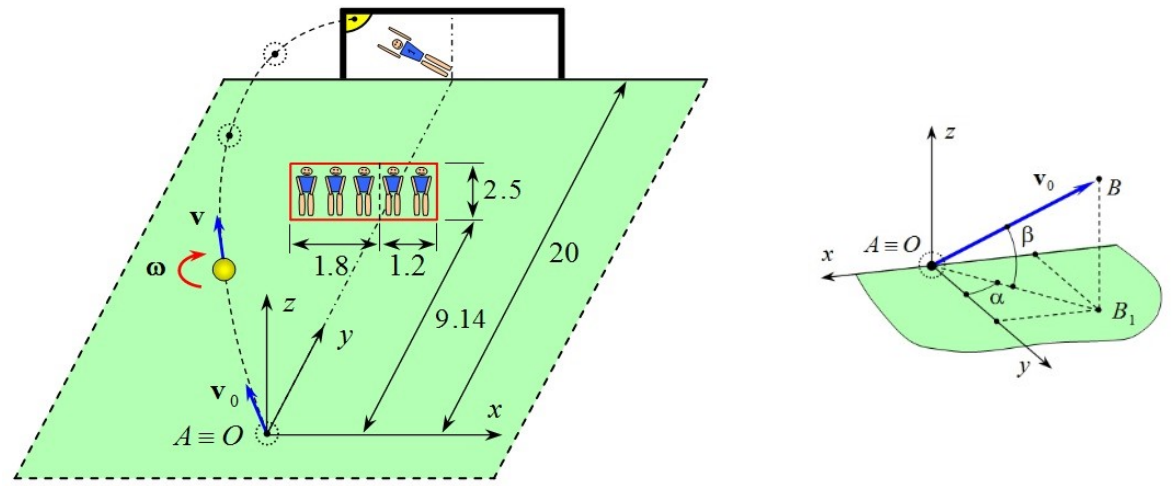

Fig. 9. Direct free kick. 
that the defenders wall is wide $3 \mathrm{~m}$ and the guarded height is $2.50 \mathrm{~m}$. The wall is positioned asymmetrically with respect to the central longitudinal line of the football field $1.2 \mathrm{~m}$ in one direction and $1.8 \mathrm{~m}$ in the other (Fig.9).

The striker, who performs the direct free kick, attempt to strike the ball with an appropriate in direction and magnitude linear velocity in order to transfer it over the wall. Simultaneously with the required linear velocity, the striker must give to the soccer ball the required angular velocity, i.e. side rotation. Namely on the base of this rotation the Magnus effect starts to act as the ball goes "turning" in its trajectory as it approaches the goal. At this deviation of the trajectory the ball is directed into the upper corner of the goal where the dangerous zone is located (Fig.9).

\section{Numerical solution}

For the numerical solution of the differential equations $(11) \div(16)$ a program in the MatLab-Simulink environment is created. As initial conditions in the program are introduced the following geometric and kinematic parameters of the ball - initial position, initial angle, initial linear and angular velocities.

By the program can be obtained all kinematic characteristics of the soccer ball motion: the lows of motion about the six coordinates $x(t), y(t), z(t), \psi(t), \theta(t), \varphi(t)$, the trajectory projections $z(x), z(y), y(x)$ on the coordinate planes $O x z, O y z, O x y$, the linear velocities $: \ldots, \ldots,: \ldots, v(t)$, the angular velocities $\vdots \ldots, i_{1}, \dot{\varphi}(t)$, $\omega_{X}(t), \omega_{Y}(t), \omega_{Z}(t), \omega(t)$, the linear accelerations ......... , $a(t)$, the angular accelerations $\because \ldots,{ }_{\sim}, \ddot{\varphi}(t), \varepsilon_{X}(t), \varepsilon_{Y}(t), \varepsilon_{Z}(t), \varepsilon(t)$.

\section{Results and discussions}

Fig. 10 shows the trajectory of a successful corner kick (CK) with outside spin to the remote target (in the right upper corner of the goal) at the initial parameters: linear velocity $v=29.54 \mathrm{~m} / \mathrm{s}$, angular velocity $\omega=97.88 \mathrm{rad} / \mathrm{s}(935 \mathrm{rpm}), \alpha=5.71^{\circ}, \beta=17.74^{\circ}$.

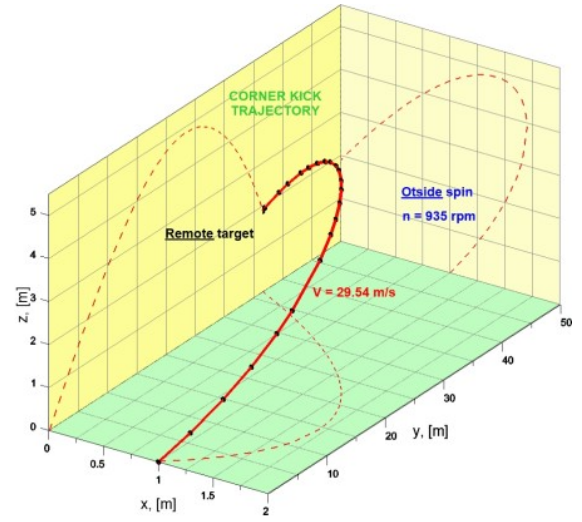

Fig. 10. Successful corner kick to remote target.

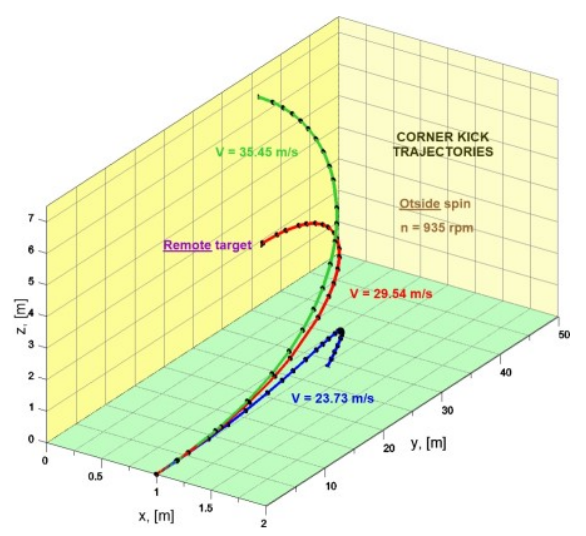

Fig. 11. Three kicks with different velocities.

On the next Fig. 11 are presented three different corner kick trajectories, which are related to: the above mentioned (see Fig. 10) successful kick (red line) and two unsuccessful (failed) ones. Both failed kicks have the same initial angular velocity and 
angles as the successful one. The linear velocity of the first of them (green line) is $v=35.45 \mathrm{~m} / \mathrm{s}$ while for the second (blue line) $v=23.73 \mathrm{~m} / \mathrm{s}$. The trajectory projections of the presented on the Fig.11 kicks are given also on Fig. 12 and Fig.13, where they are drawn in the planes $y z$ and $x z$, respectively. It can be concluded that at the lower linear velocity (blue dotted line) the ball can't reach at all to the goal while with higher velocity (green dotted line) the soccer ball goes very high and away from the goal target.

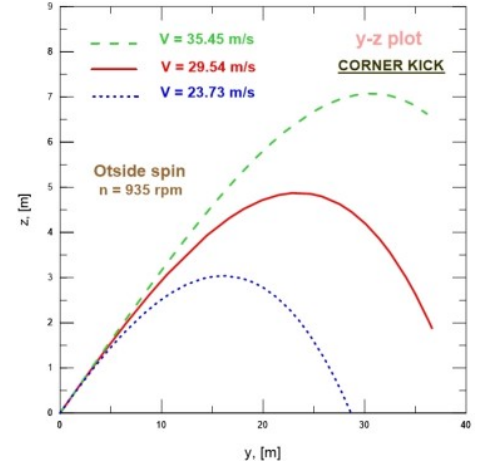

Fig. 12. Trajectory projections of three different corner kicks in $y z$ plane.

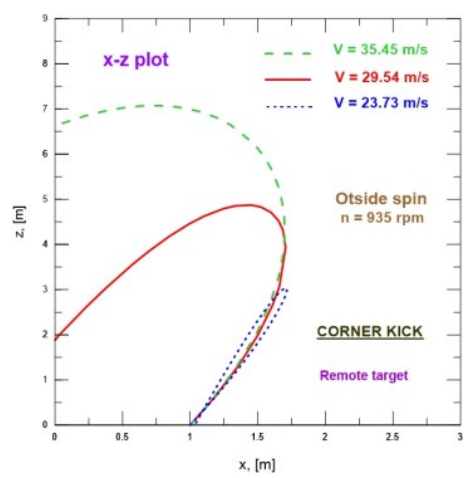

Fig. 13. Trajectory projections of three different corner kicks in $x z$ plane.

The trajectories of two successful free kicks (FK) to the left (green line) and right (red line) target (left and right upper corners of the goal) are shown on Fig. 14. Both of them are realized with outside spin. The initial parameters are: linear velocity $v=24.15 \mathrm{~m} / \mathrm{s}$, angular velocity $\omega=61.86 \mathrm{rad} / \mathrm{s}(591 \mathrm{rpm}), \alpha=15.74^{\circ}, \beta=18.84^{\circ}$.

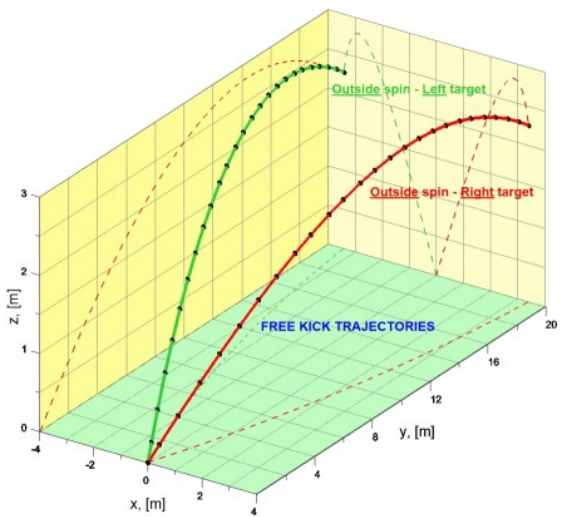

Fig. 14. Outside spin to left \& right target.

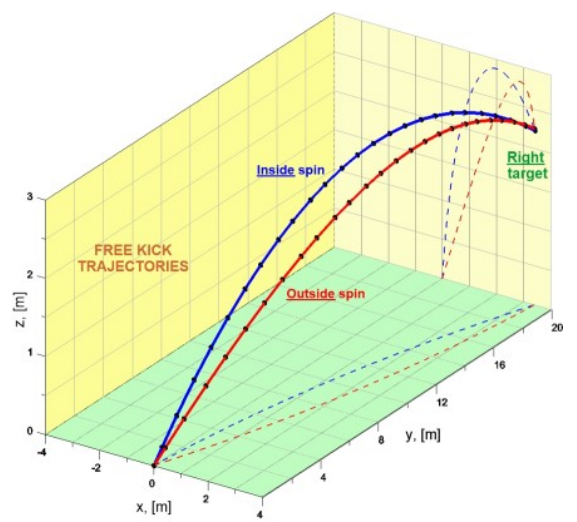

Fig. 15. Outside \& inside spin to right target.

On the next Fig. 15 are presented the trajectories of two successful free kicks to the right target of the goal. The first one is realized with outside spin (red line) at the same as above-mentioned initial angular velocity and angles. The second of them is related to inside spin (blue line) at the following initial parameters: linear velocity $v=23.42 \mathrm{~m} / \mathrm{s}$, angular velocity $\omega=58.95 \mathrm{rad} / \mathrm{s}(563 \mathrm{rpm}), \alpha=3.64^{\circ}, \beta=19.72^{\circ}$.

The complex solution of finding the trajectories of four possible successful free kicks, respectively to the left and right targets with outside and inside spins, are illustrated on Fig. 16. The situated on the right side two free kicks are already described and they are the same 
as shown on Fig. 15. The other two free kicks are directed to the left target, as they are also realized with inside and outside spin. For them it would be specially mentioned that they are symmetrical related to the first pair of free kicks on the right side.

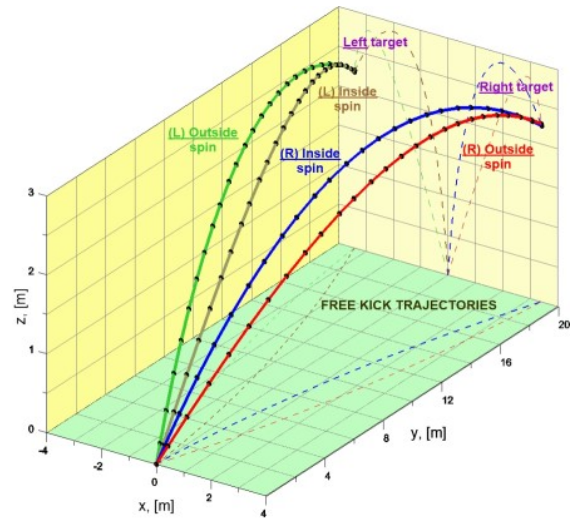

Fig. 16. Outside and inside spins to both targets.

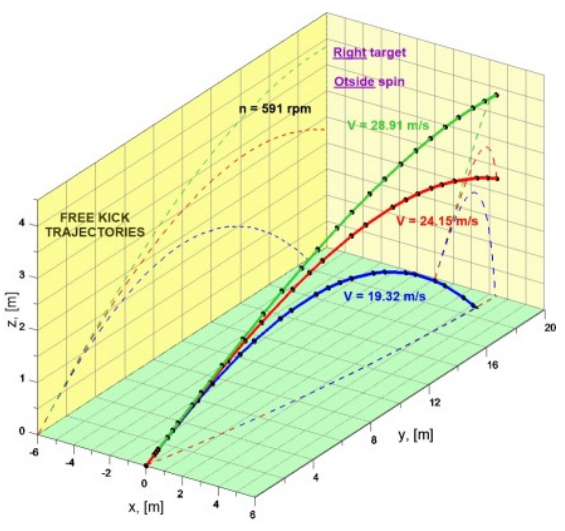

Fig. 17. Three different kicks with outside spin to right target.

On the next Fig. 17 are presented three different free kick trajectories to the right target. The first one is successful (red line) and it is done at the same initial conditions as the above-mentioned ones (see Fig. 14). Both other free kicks are unsuccessful, as their initial angular velocity and angles are the same as for the successful one. The linear velocity of the first failed kick (green line) is $v=28.91 \mathrm{~m} / \mathrm{s}$ as for the second failed kick (blue line) this velocity is $v=19.32 \mathrm{~m} / \mathrm{s}$.

From the numerical results it is observed as general that a soccer ball can reach the target only under proper values of the initial conditions. These are correctly defined direction and magnitude of the linear velocity also the corresponding angular velocity. So, at proper initial conditions and on the base of the Magnus effect, the trajectory of the soccer ball deviates in the appropriated direction, as the ball goes to the target of the goal. The initial conditions of the successful kicks in fact are limited to a very small range, so only the best football players, thanks to their skills and experience, can realize them. By the obtained results it can be seen that corner or free kicks, which are done with very low or very high values of initial linear velocities are unsuccessful.

\section{Conclusions}

In this paper a study from the mechanical point of view of the most important kicks in the football game is presented. These are the corner and direct free kick.

The characteristics of soccer ball size five are assumed and the latter is considered as an ideal rigid sphere. The orientation of the ball in the space is defined by Cardan angles. The aerodynamics forces acting to the ball during its flight are taken into account. The system of six nonlinear equations is composed.

Furthermore, in relation with a modelling of the goal target, two of the most dangerous zones of the goal are defined as well as a short description of the geometry and implementations of both investigated soccer kicks is presented.

The numerical solution of the problem is carried out by a program in the MatLabSimulink environment. All possible kinematic characteristics of the soccer ball flight, as lows of motion, linear and angular velocities, linear and angular accelerations and trajectories are obtained. 
The effects of several initial parameters, as velocities and angles, on the ball flight in the football game are studied. Based on the analysis of the results, the recommended values of initial parameters for successful corner and free kicks are found. All of the trajectories are illustrated by $3 \mathrm{D}$ or $2 \mathrm{D}$ graphics.

The study is suitable and useful for scientists in the field of sport engineering as well as for soccer trainers.

\section{References}

1. R. Cross, Am. J. Phys. 70(5), 482-489 (2002)

2. R. D. Mehta, J. M. Pallis, Materials and Science in Sports, 185-197 (2001)

3. A. M. Nathan, Am. J. Phys. 76(2), 119-124 (2008)

4. P. Jalilian, P. K. Kreun, M. M. Makhmalbaf, W. W. Liou, Am. J. Sport Sc. 2(5), $115-$ 121 (2014)

5. J. Leela, D. M. G. Comissiong, K. Rahaman, Lat. Am. J. Phys. Educ. 8(4), 4505-14505-9 (2014)

6. A. Ivanov, J. Javorova, Tehnomus J. 24, 54-61 (2017)

7. T. Asai, M. J. Carre, T. Akatsuka, S. Haake, Sports Eng. 5, 183-192 (2002)

8. T. Asai, T. Akatsuka, S. Haake, Phys. World 11 (6), 25-27 (1998)

9. M. J. Carre, T. Asai, T. Akatsuka, S. Haake, Sports Eng. 5, 193-200 (2002)

10. T. Asai, K. Seo, O. Kobayashy, R. Sakashita, Sports Eng. 10, 101-110 (2007)

11. J. Kim, H. Park, H. Choi, J.Y. Yoo, J. Fluid Mech. 754, R2, doi:10.1017/jfm.2014.428 (2014)

12. T. Yamagata, T. Nagasawa, N. Fujisawa, T. Asai, J. Flow Control, Measur. \& Visual. 1, 86-93 (2013)

13. J. E. Goff, M. J. Carre, Am. J. Phys. 77(11), 1020-1027 (2009)

14. J. E. Goff, M. J. Carre, Eur. J. Phys. 31, 775-784 (2010)

15. L. Oggiano, L. Saetran, Procedia Eng. 2, 2473-2479 (2010)

16. J. E. Goff, J. Kelley, C. M. Hobson, K. Seo, T. Asai, S. B. Choppin, Eur. J. Phys. 38, 044003 (2017)

17. T. Asai, S. Ito, K. Seo, S. Koike, Procedia Eng. 34, 122-127 (2012)

18. D. Rogers, M.A. Passmore, A. Harland, R. Jones, C. Holmes, T. Lucas, Procedia Eng., Elsevier 2(2), 2481-2486 (2010)

19. J. C. Cooke, The mathematical Gazette, 39, 196-199 (1955)

20. S. Verma, Int. J. Scientific Research 4(1), 420-424 (2015)

21. F. Reid, J. Parabola, 33(1), 1-4 (1997)

22. K. E. Kenyon, Natural Science 8, 49-52 (2016).

23. K. Bray, D. G. Kerwin. J. Sports Science 21, 75-85 (2003)

24. M. J. Carre, S. R. Goodwill, S.J. Haake, Proc. IMechE Part C: J Mech Eng Sci, 219(7), 657-666 (2005)

25. B. G. Cook, J. E. Goff, European J. Physics 27, 865-874 (2006)

26. J. E. Goff, Physics Today, 63(7), 62-63 (2010)

27. A.M. Alcock, W. Gilleard, A.B. Hunter, J. Baker, N. Brown, J Sports Sci 30(4), 387394 (2012)

28. A. Katis, E. Kellis, A. Lees, Sports Biomech. 14 (3), 287-299 (2015) 